\title{
Head and neck mycobacterial infections in pediatric patients
}

\author{
G. Caruso ${ }^{\text {a, }}$, F.M. Passàli $^{b}$, L. Salerni $^{\text {a }}$, G. Molinaro ${ }^{c}$, M. Messina $^{c}$ \\ ${ }^{a}$ ENT Department, University of Siena, Italy \\ ${ }^{\mathrm{b}}$ ENT Department, University of Tor Vergata, Rome, Italy \\ 'Pediatric Surgery Unit, University of Siena, Italy
}

\section{A R T I C L E I N F O}

\section{Keywords:}

Pediatric mycobacterial infection

Cervical lymphadenopathy

Surgical treatment

\begin{abstract}
A B S T R A C T
Objective: Lymph nodal disease is one of the most common manifestations of head and neck tuberculosis and is particularly frequent in paediatric patients with an increasing incidence in the last decade. It may represent the manifestation of a systemic tuberculous disease or a clinical entity specific of the neck. Aim of this paper is to retrospectively analyse mycobacterial cervical adenopathies observed in two Paediatric European Centers between 1986 and 2004 and the outcomes of medical or surgical treatment.

Methods: 353 children were examined for mycobacterial cervical lymphadenopaties since January 1986 to December 2004. Demografic data about the sample are showed. The retrospective evaluation of the sample underlined distribution according to etiologic patterns, head and neck adenopathies localization. Previous or simultaneous medical or surgical treatment were analyzed. Statistical analysis with Chi Square test was performed.

Results: 281 (79.60\%) cases showed a higher localization and 72 (20.40\%) a lower localization. In relation to the etiological agent, 8 (2.27\%) Mycobacterium tuberculosis (MTB) and 222 (62.89\%) mycobacteria other than tuberculosis (MOTT) lymphadenopaties were observed in the upper localization as opposed to 21 (5.95\%) MTB and $39(11.05 \%)$ MOTT in the lower plane. Two (0.56\%) were detected as upper lymph nodal tumefaction in the median line. In 86 (24.36\%) cases at the MRI the so-called "iceberg effect" was noted. 163 (62.45\%) patients underwent primary excisional biopsy whereas $74(28.35 \%)$ underwent exeresis after other unsuccessfull therapies, 9 underwent only drainage, and 15 drainage with subsequent antibiotic therapy. Number of relapses after surgery was 16 (6.13\%).

Conclusions: A not homogeneous therapeutic approach to the mycobacterial cervical adenitis arises from literature and WHO guidelines does not give indications for the treatment of the cervical pattern.

A therapeutic strategy based on the etiology is mandatory. In case of MBT adenopathy the therapy of choice includes the association, in variable way, of different chemotherapic drugs; surgery is reserved to advanced cases. Conversely, in cases of MOTT adenopathy, surgery is the treatment of choice.
\end{abstract}

(c) 2009 Elsevier Ireland Ltd. All rights reserved.

\section{Contents}

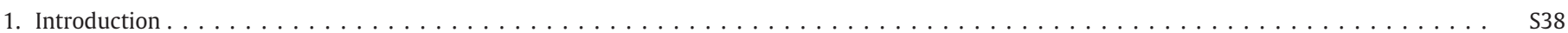

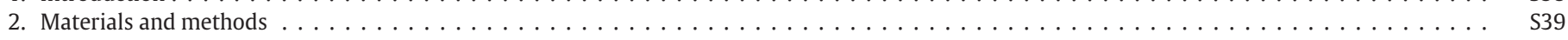

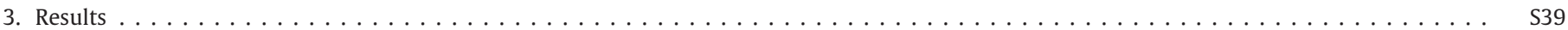

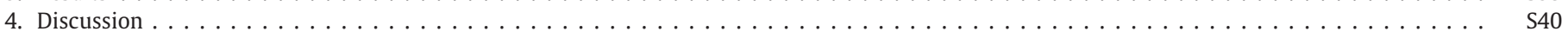

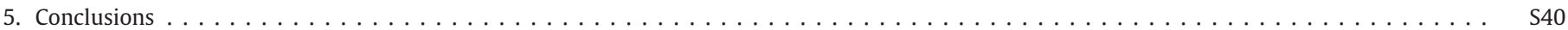

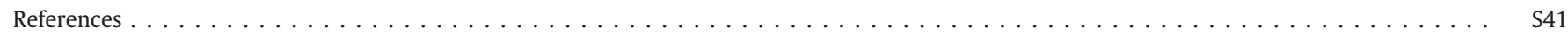

\section{Introduction}

Tuberculosis (TB) is usually considered an infectious disease whose multiple etiologic agents are commonly classified into two

\footnotetext{
* Corresponding author: Dr. Giuseppe Caruso, Clinica Otorinolaringoiatrica, Policlinico Universitario "Le Scotte", Viale Bracci 16, 53100 Siena, Italy. Tel.: +39 0577585470

E-mail address: dr.gcaruso2002@virgilio.it (G. Caruso).
}

large groups: Mycobacterium tuberculosis (MTB) and mycobacteria other than tuberculosis (MOTT) also called atypical mycobacteria (AM). In children, the principal causative agents are AM in $92 \%$ of cases, while MTB is involved in the rest of cases [1]. It has been estimated that $65 \%$ to $80 \%$ of children under 12 years are infected by Mycobacterium avium complex; $10 \%$ to $20 \%$ by Mycobacterium scrofulaceum; and $10 \%$ by MT. On the other hand, in paediatric patients aged more than 12 years MT is the principal etiologic agent. Incidence and prevalence of pediatric tuberculosis 
worldwide has been estimated around 3.1 million children under 15 years of age [2]: the majority of cases occur in countries where the prevalence HIV/AIDS is high. TB occurs frequently among disadvantaged populations, such as malnourished individuals, and those living in crowded areas [3]. TB cases are once again frequent in low socio-economic urban areas and among different ethnic groups. A fair percentage (25\%) is composed by immigrant children from endemic areas who, together with suckling and post-puberal teenagers, appear to be at a higher risk of infection [4]. It is important that pediatric otolaryngologists are aware of tubercular infection of head and neck with its different, varied and sometimes unusual presentations [5] not only in third world countries but also in industrialized areas. TB is an airborne disease, transmitted by Pflugge particles expelled by subjects sneezing, coughing, speaking or singing with pulmonary or laryngeal TB. The source of infection in cervical lymph nodes is controversial. The initial tubercular infection starts in the tonsillar crypts and hence diffuse to the cervical lymph glands.

Lymph nodal disease is one of the most common manifestations of extra-pulmonary tuberculosis and is particularly frequent in paediatric patients. TB involving cervical lymph nodes represents the $50 \%$ of extrapulmonary TB [6].

In the neck the posterior, the anterior and supraclavicular cervical regions are usually involved. The submandibular, submental, axillary, and inguinal lymph nodes are less frequently affected. The involved lymph nodes are typically firm, non-tender, and painless, with non-erythematous overlying skin. They are initially non-fluctuant: lymph node suppuration and spontaneous drainage may occur after caseation and necrosis development [7,8]. Fever, weight loss, fatigue, and malaise are usually absent or minimal. Lymph node involvement typically occurs between six to nine months following the initial infection.

Diagnosis is not always simple and sometimes only a "plausible" diagnosis of mycobacterial lymphadenopathy is formulated, based on positive skin reactivity tests, a highly suggestive clinical course and histological findings with acid-alcohol resistant bacteria with impossibility of isolating the microorganism. Actually, mainly thanks to PCR, the identifications of bacteria is almost always possible. Furthermore, differential diagnosis of cervical swelling in paediatric patients is extremely demanding as this comprises several conditions which cannot be easily distinguished [9]. It emerge that no diagnostic-therapeutical gold standard has been set as far as mycobacterial cervical lymphadenopathy is concerned. Several therapies are attempted, each of them with valid application criteria and presenting contraindications.

Aim of this paper is to retrospectively analyse mycobacterial cervical adenopathies observed in two Paediatric European Centers between 1986 and 2004. Of these cases, treatment modalities and outcome have been evaluated over time.

\section{Materials and methods}

At the Paediatric Surgery Unit of the Department of Paediatrics, Obstetrics and Reproductive Medicine of the University of Siena (Italy) and the Paediatric Surgery Unit of the Maternal-Paediatric Department of the Hospital University Vall d'Hebron in Barcelona (Spain) between January 1986 and December 2004, 353 children were examined for mycobacterial cervical lymphadenopaties. Inclusion criteria in the study were "incontrovertible" isolation and identification of mycobacteria in the cervical lesion, or at least evidence of acid alcohol resistant bacteria in the involved tissues.

Patients were 142 males (40.22\%) and 211 females (59.78\%), age ranging between 1 month and 16 years. All subjects were examined at 10 to 14 months from the cervical onset. Two hundred forty three patients $(68.83 \%)$ were already undergone a wide spectrum antibiotic therapy for at least 2 weeks with no clinical improvement.
Table 1

Distribution according to sex and other previous or simultaneous therapy

\begin{tabular}{ll}
\hline$N=353$ & $142 \mathrm{M}(40.22 \%)$ \\
& $211 \mathrm{~F}(59.78 \%)$ \\
$-243(68.83 \%)$ & previous wide spectrum antibiotic therapy \\
$-\quad 17(4.81 \%)$ & chemotherapy for malignancies \\
$-\quad 5(1.4 \%)$ & immunosuppressive therapy \\
\hline
\end{tabular}

Table 2

Distribution according to treatment

\begin{tabular}{cc}
\hline Group A: 311 primary surgery & 218 excisional biopsy \\
$(88.1 \%)$ & 69 drainage and exeresis \\
& 24 drainage \\
Group B: 42 specific antibiotoc treatment \\
$(11.9 \%)$
\end{tabular}

Out of 353 children in the study, 3 (0.84\%) were undergone liver transplantation and $2(0.56 \%)$ kidney transplantation: in both of them anti-rejection immunosuppressive therapy was being administered; 17 (4.81\%) were contemporarily undergoing chemotherapy for different malignancies. Of them only $2(0.56 \%)$ had completed chemotherapy 6 weeks and 5 months prior, respectively; 2 (0.56\%) were HIV positive and 8 (2.27\%) showed active AIDS, 2 of these patients had been previously diagnosed with abdominal mycobacterial lymphonodal infection (Table 1).

All patients underwent clinical evaluation and tubercoline reactivity skin tests, ultrasonography and neck Magnetic Resonance Imaging (MRI).

A subclassification of the patients was made regarding the localization (above or below the plane of the hyoid bone) and the etiologic agent. The subjects were also subdivided into two groups on the basis of treatment received: group A 311 (88.10\%) patients submitted to primary surgery, 218 (61.76\%) of them had primary excisional biopsy, 24 (6.80\%) drainage, 69 (19.55\%) drainage followed by excisional biopsy; and group B 42 (11.9\%) patients submitted to specific antibiotic therapy prior to surgery, based on the results of skin reactivity tests (Table 2).

Surgically removed lymph nodes underwent Ziehl-Neelsen staining, and histopathologic examination.

In 103 cases $(29.18 \%)$ the surgery was followed by medical antitubercolar therapy, usually when skin results were unequivocal and a "cross reactivity" was observed or when a clinical negative neck was only achieved by a "safety" pharmacological support.

Results were compared by means of Square chi test with a significance at $p \leq 0.05$.

\section{Results}

281 cases (79.60\%) showed a higher localization of adenophaties (above the hyoid bone) and $72(20.40 \%)$ a lower localization. In relation to the aetiological agent 8 (2.27\%) MTB lymph adenopaties and 222 MOTT (62.89\%) were observed in the upper localization as opposed to 21 MTB (5.95\%) and 39 MOTT (11.05\%) in the lower plane. Two cases were detected $(0.56 \%)$ of upper lymph nodal tumefaction in the median line which placed a differential diagnostic doubt with a cysts of the thyroglossal duct.

In 86 cases (24.36\%) at the MRI the so-called "iceberg effect" was noted: the lesions appeared small upon palpation and enough mobile on the surface but during the surgery they revealed a 5-6 times larger volume than expected and firm adherence to the deep neck structures. All the cases in the study with no exception, showed at least the presence of acid-alcohol fast bacilli in the cervical lesion. However, in the noticeable percentage of $17.84 \%$ (63 children) the bacillus could not be isolated and positively identified.

Out of 290 identified cases, 23 (7.93\%) were affected by MTB and $6(2.06 \%)$ by $M$. bovis. Of these, 19 underwent antibiotic therapy 
Table 3

Distribution according to etiologic agents

Mycobacteria other than tubercolosis (MOTT)

Mycobacterium tubercolosis (MBT)

Not otherwise specified (NOS)
261

63 with 12 recovery and 7 relapses treated subsequently with surgery. Ten pediatric patients underwent surgery as first option: in 6 cases a drainage was associated to antibiotic therapy with no relapse, in the other 4 cases affected by large and fistulized neck lesions a successful complete surgical excision was performed.

In 261 cases (90\%) an unequivocal diagnosis of MOTT infection was expressed and respectively in 163 cases (62.45\%) an intracellular M. avium was detected, in 39 cases (14.94\%) a M. scrofulaceoum, in 19 cases (7.27\%) a M. fortuitum, in 36 cases ( $13.79 \%$ ) a M. kansasii, in 2 cases $(0.76 \%)$ a $M$. xenotopi, in 1 case $(0.38 \%)$ a $M$. genavensis and finally in 1 case $(0.38 \%)$ a M. simiae (Table 3 ).

No patient with MOTT cervical adenopathy recovered with exclusive antibiotic therapy. One hundred sixty three patients (62.45\%) underwent primary excisional biopsy, whereas 74 (28.35\%) underwent exeresis after other unsuccessful therapies, 9 underwent only drainage, and 15 drainage with subsequent antibiotic therapy: of these respectively 8 and 12 pediatric patients relapsed and were treated subsequently with a complete exeresis. The number of relapses after surgery was 16 (6.13\%).

The statistical analysis of data shows that in cases of MOTT cervical adenopathies, the efficacy of surgery is higher than that of pharmacological therapy $(p<0.01)$ and that among different surgical options exeresis is more effective that the drainage alone $(p<0.01)$.

On the contrary, in lymph nodal infection due to MTB, antibiotic therapy is as effective as surgery in case of small and non fistulized lesions $(p<0.01)$. Conversely, in large, colliquated lesions, or in lesions with skin fistulae, surgery is always required $(p<0.01)$ since the healing rate is exceedingly higher. In this case as well, the efficacy of complete exeresis proved to be higher than that of drainage $(p<0.05)$.

\section{Discussion}

Mycobacterial lymphadenopathies are scarcely invasive and easily identifiable; however a differential diagnosis from MOTT and MTB should be made, mainly because the treatment of both is thoroughly different [10].

Clinical presentation of MOTT lymphadenopathies often overlaps with that of MBT, though these generally evolve with a less severe pattern. The most frequently affected subjects are children under 5 years of age, showing an adenopathy of the upper cervical nodes resistant to a 10-15 days large spectrum antibiotic treatment.

After 3-4 weeks, the swelling considerably grows with redness of the skin and an extremely disagreeable appearance. If a MTB infection is mistakenly diagnosed, as it frequently happens, antibiotics will fail with consequent further enlargement and fluctuation of the lesion up to fistulization [11].

In our case series patients, diagnosis of MOTT adenopathy was made in $75.9 \%$ of cases in children from 0 to 6 years of age; in $17.9 \%$ of a cases ranging between 6 and 12 years and in $6.2 \%$ of cases ranging between 12 and 16 years. An inverted incidence is usually recorded in patients with MTB adenopathy.

The diagnostic path includes intra-dermal reactions [12]. These skin tests performed in all our patients for the detection of MTB and in 277 patients for the detection of MOTT, have shown highly effective and reliable and were confirmed by the isolation of the corresponding Mycobacterium in $73.9 \%$ of cases. Their application allowed a rational treatment even while waiting for the microbio- logical results which usually take $8-12$ weeks to be obtained, even if the diagnostic gold standard is still the culture of mycobacteria.

The subject's immunogical reactivity is very important in case of mycobacterial lymphadenopathy: the severity of mycobacterial lymphoadenitis in HIV positive patients is extensively described in the literature $[13,14]$.

Our data clearly show that in children with primary immunodepression of any kind, or undergoing chemotherapy or being administered post-transplantation immunosuppressive therapies, lesions always rapidly grow and rapidly spread. The histopathological pattern is also typical in subjects with immunodeficiency; in none of these patient either colliquation or caseous granulomas were observed. For this reason, a prompt therapeutic or surgical approach is mandatory as well as an evaluation of the immunological pattern. As concerns therapeutic strategy, the analysis of the collected data clearly show a difference between a MOTT infection and one caused by "typical" bacteria. In MBT lymphadenopathies, medical management proved effective with a more than acceptable success rate, though surgery is still mandatory in case of fistulized lesions [15]. Excisional biopsy may be immediately necessary in cases of bulky and advanced lesions as antibiotics have a limited efficacy in metabolically inert and scarcely vascularized lesions. An elective surgery should be postponed in patients with suspected active TB. In any case, major surgical procedures as selective neck dissection should be avoided [16].

On the contrary, antibiotics are ineffective in MOTT lesions and cannot even induce a regression of very limited and initial patterns. In a relevant percentage of cases, while waiting for the efficacy of drugs to be assessed, lesions may deteriorate and the following intervention may become complicated [17]. In our case series, the success rate was much higher with a primary surgical approach (92.3\%) and the surgery of choice was a complete exeresis. When this is not applicable, surgical drainage can be performed and area courettage, although these treatments are still affected by an extremely high recurrence rate (up to $75 \%$ ). On the contrary, post-exeresis recurrence rate is very low $(6.1 \%)$.

A special reference has to be made to evaluate the aesthetic outcome over time following surgery. Out of 353 cases examined, only 4 patients developed keloid or pathological scar, all following very bulky and very skin infiltrating lesions. All the 4 cases showed a successful scar removal.

\section{Conclusions}

Cervical adenopathies in children represent a major challenge to the Paediatrician and Paediatric Otorhinolaryngologist. The diagnostic chances in the neck are several and the difference must be highlighted between congenital and acquired tumoral and infectious masses. A review of the Literature shows a globally non homogeneous therapeutic approach to the mycobacterial cervical adenitis [18]. The WHO indicates guidelines to control childhood tuberculosis for the prevention of TB, but does not give indications for the treatment of the cervical pattern [19].

In our opinion, the most rational approach is to define a therapeutic strategy based on the etiology. In case of MBT adenopathy the therapy of choice includes the association in variable way of a different chemotherapic drugs and surgery in advanced cases (fistulae or relapses). Conversely, in cases of MOTT adenopathy, the surgery is the treatment of choice. In these case, the radicality of surgery is closely related to prognosis, whereas either administration or not of chemotherapic drugs does not seem to be determinant to recovery.

\section{Conflict of interest}

None declared. 


\section{References}

[1] Powell DA, Hunt WG. Tuberculosis in children: an update. Adv Pediatr 2006;53:279-322.

[2] Morcillo N, Zumarraga M, Imperiale B, Di Giulio B, Chirico C, Kuriger A, Alito A, Kremer K, Cataldi A. Tuberculosis transmission of predominant genotypes of Mycobacterium tuberculosis in northern suburbs of Buenos Aires city region. Rev Argent Microbiol 2007;39:145-150.

[3] Starke JR. New concepts in childhood tuberculosis. Curr Opin Pediatr 2007;19(3):306-313.

[4] Stout JE, Ostbye T, Walter EB, Hamilton CD. Tuberculosis knowledge and attitudes among physicians who treat young children in North Carolina, USA. Int J Tuberc Lung Dis 2006;10:783-788.

[5] Prasad R. Management of multi-drug resistant tuberculosis: practitioner's view point. Indian J Tuberc 2007;54:3-11

[6] Lenci G, Gartenschlager M. Current aspects of lymph node tuberculosis of the neck. Pneumologie 1996;50:426-428.

[7] Cruz AT, Starke JR.Clinical manifestations of tuberculosis in children. Paediatr Respir Rev 2007;8:107-117.

[8] Freixinet J, Varela A, Lopez Rivero L, Caminero JA, Rodríguez de Castro F, Serrano A. Surgical treatment of childhood mediastinal tuberculous lymphadenitis. Ann Thorac Surg 1995;59:644-646

[9] Kamath MP, Bhojwani K, Naik R, Kumar R, Chakravarthy Y. Tuberculosis mimicking Kikuchi's disease. Ear Nose Throat J 2006;85:126-128.

[10] Kanlikama M, Mumbuc S, Bayazit Y, Sirikci A. Management strategy of mycobacterial cervical lymphadenitis. J Laryngol Otol 2000;114:274-278.
[11] Albright JT, Pransky SM. Non tuberculous mycobacterial infections of head and neck. Pediatr Clin N Am 2003;50:503-514.

[12] Cinar F, Cinar S, Yimax B, Gursel O. Purified protein derivative: the vital part of the cervical tuberculous adenitis dignosis. Otolaryngol Head Neck Surg 2003;129:245-247.

[13] Mfinanga SG, Morkve O, Kazwala RR, Cleaveland S, Sharp MJ, Kunda J et al. Mycobacterial adenitis: role of Mycobacterium bovis, non-tuberculous mycobacteria, HIV infection, and risk factors in Arusha, Tanzania. East Afr Med J 2004;81(4):171-178.

[14] Mtei L, Matee M, Herfort O, Bakari M, Horsburgh CR, Waddell R et al. High rates of clinical and subclinical tuberculosis among HIV-infected ambulatory subjects in Tanzania. Clin Infect Dis 2005;40(10);1500-1507.

[15] Messina M, Carfagna L, Meucci D, Ferrucci E, Tallarico R. Linfoadeniti cervicali da Micobatterio. Minerva Chir 2000;55(12):847-853.

[16] Ammari FF, Bani Hani AH, Ghariebeh KI Tuberculosis of the lymph glands of the neck: A limited role for surgery. Otolaryngol Head Neck Surg 2003;128:576-580.

[17] Stewart MG, Starke JR, Coker NJ. Nontuberculous mycobacterial infections of the head and neck. Arch Otolaryngol Head Neck Surg 1994;120:873-876.

[18] Tunkel DE, Romaneschi B. Surgical treatment of cervicofacial nontuberculous mycobacterial adenitis in children. Laryngoscope 1995;105:1024-1028.

[19] Sant'Anna CC, Hijjar MA. Recent contribution of the World Health Organization to control childhood tuberculosis. Rev Saude Publica 2007;41(Suppl 1):117-120. 